\title{
On the Strong Property of Connected Open-Close and Close-Open Filters
}

\author{
Jose Crespo $^{1}$, Victor Maojo ${ }^{1}$, José A. Sanandrés ${ }^{1}$, Holger Billhardt ${ }^{1}$, and \\ Alberto Muñoz ${ }^{2}$ \\ 1 Laboratorio de Inteligencia Artificial \\ Facultad de Informática \\ Universidad Politécnica de Madrid \\ 28660 Boadilla del Monte (Madrid), Spain \\ jcrespo@fi.upm.es \\ 2 Departamento de Radiodiagnóstico \\ Hospital 12 de Octubre \\ Ctra. Andalucia Km. 5'400 \\ 28041 Madrid
}

\begin{abstract}
This paper studies connectivity aspects that arise in image operators that process connected components of an input image. The focus is on morphological image analysis (i.e., on increasing image operators), and, in particular, on a robustness property satisfied by certain morphological filters that is denominated the strong-property. The behavior of alternating compositions of openings and closings will be investigated under certain assumptions, especially using a connected component preserving equation. A significant result is the finding that such an equation cannot guarantee the strong property of certain connected alternating filters. The class of openings and closings by reconstruction should therefore be defined to avoid such situations.
\end{abstract}

\section{Introduction}

This paper studies the strong property of morphological filters that satisfy certain conditions regarding connectivity. The strong property is a robustness property introduced in the morphological filtering framework [1] [2. Morphological filters that satisfy this interesting property are more robust to small variations of the input image, such as noise, under certain limits.

The strong property is somewhat related to connectivity issues in the sense that some morphological filter types that do not satisfy the strong-property condition are in fact strong when they are connected. In this work we will study this property for connected filters that satisfy different requirements. A central point will be to study connected filters that satisfy a well-known additional condition that not only the filter output is invariant but also each of its connected components.

In this paper we will obtain a significant result concerning the strong property of alternating filters when such a condition is used to define them, in the sense

A. Braquelaire, J.-O. Lachaud, and A. Vialard (Eds.): DGCI 2002, LNCS 2301, pp. 165 174 2002. (C) Springer-Verlag Berlin Heidelberg 2002 
that the fact that an alternating filter is strong does not imply that the dual family under that definition is strong as well. Of course, it is not the case that the result contradicts the morphological duality principle; instead, the issue is that the condition does not treat symmetrically (and dually) openings and closings. We therefore define the class of openings and closings by reconstruction in a way to avoid such a problem. Those definitions have been used previously by the authors of this paper, and this work strongly supports that choice.

The outline of the paper is as follows. Section 2 provides some background on mathematical morphology, in which some concepts concerning connectivity are commented. Then, Section 3 studies the strong property and the behavior of connected openings, closings, and alternating filters that satisfy certain conditions. An important result showing certain problems motivates the definition of openings and closings by reconstruction to avoid them. Finally, a conclusion section ends the paper.

\section{Some Concepts and Definitions}

\subsection{Basic Notions}

Mathematical morphology concerns the application of set theory concepts to image analysis. General references are [3] [1] [2] [4].

A basic set of notions on mathematical morphology can be the following:

- Mathematical morphology deals with increasing mappings defined on a complete latice [5] [2]. In a complete lattice there exists an ordering relation, and two basic operations called infimum and supremum (denoted by $\wedge$ and $\bigvee$, respectively).

- A transformation $\psi$ is increasing if and only if it preserves ordering.

- A transformation $\psi$ is idempotent if and only if $\psi \psi=\psi$.

- A transformation $\psi$ is a morphological filter if and only if it is increasing and idempotent.

- An opening (often denoted by $\gamma$ ) is an antiextensive morphological filter.

- A closing (often denoted by $\varphi$ ) is an extensive morphological filter.

In all theoretical expressions in this paper, we will be working on the lattice $\mathcal{P}(E)$, where $E$ is a given set of points called space and $\mathcal{P}(E)$ denotes the set of all subsets of $E$ (i.e., $\mathcal{P}(E)=\{A: A \subseteq E\}$ ). In other words, inputs and outputs will be supposed to be sets or, equivalently, binary functions. In this lattice, the sup $\bigvee$ and the inf $\bigwedge$ operations are the set union $\bigcup$ and the set intersection $\bigcap$ operations, while the order relation is the set inclusion relation $\subseteq$. Even though we will work on the lattice $\mathcal{P}(E)$, results are extendable for gray-level functions by means of the so called flat operators [6] [1].

\subsection{The Point Opening $\gamma_{x}$ : The Connected Component Extraction Operator}

Let us assume that the space $E$ is provided with a definition of connectivity. For all pairs of points $x, y$ in $E$, it is possible to establish whether they are connected 
or not. For example, when the space of points $E$ is $\mathbf{R}^{2}$ or $\mathbf{Z}^{2}$ (associated with the usual connectivity), a pair of points $x, y$ in a set $A$ is said to be connected if there exists a path linking $x$ and $y$ that is also included in $A$.

Connectivity is established more generally in [2] by means of the connected class concept. A connected class $\mathcal{C}$ in $\mathcal{P}(E)$ is a subset of $\mathcal{P}(E)$ such that (a) $\emptyset \in \mathcal{C}$ and for all $x \in E,\{x\} \in \mathcal{C}$; and (b) for each family $C_{i}$ in $\mathcal{C}, \bigwedge_{i} C_{i} \neq \emptyset$ implies $\bigvee_{i} C_{i} \in \mathcal{C}$. No definition of neighborhood relationships (i.e., no particular topology) has been assumed for $E$ in the definition of the connected class $\mathcal{C}$.

The subclass $\mathcal{C}_{x}$ that has all members of $\mathcal{C}$ that contain $x$ (i.e., $\mathcal{C}_{x}=\{C \in$ $\mathcal{C}: x \in C\}$ ) defines an opening called point opening 2]. The point opening of a point $x$, denoted by $\gamma_{x}$, has as invariant class (i.e., the class formed by those sets that are left unchanged by $\left.\gamma_{x}\right) \mathcal{C}_{x} \cup\{\emptyset\}$. For all $x \in E, A \in \mathcal{P}(E)$

$$
\gamma_{x}(A)=\bigvee\left\{C: C \in \mathcal{C}_{x}, C \leq A\right\}
$$

The operation $\gamma_{x}$ is therefore idempotent (i.e., $\gamma_{x}\left(\gamma_{x}(A)\right)=\gamma_{x}(A)$ or, equivalently, $\gamma_{x} \gamma_{x}=\gamma_{x}$ ) and antiextensive (i.e., $\gamma_{x}(A) \leq A$ or, equivalently, $\gamma_{x} \leq I$ ).

When we associate, for example, the operation $\gamma_{x}$ with the usual connectivity in $\mathbf{Z}^{2}$, the opening $\gamma_{x}(A), A \in \mathcal{P}\left(\mathbf{Z}^{2}\right)$, can be defined as the union of all paths that contain $x$ and that are included in $A$. Figure 1 shows an example of the result of $\gamma_{x}(A)$ where the set $A$ comprises the black regions (two connencted components or grains) and $x$ belongs to a connected component of $A$.

When a space $E$ is equipped with the opening $\gamma_{x}$, connectivity issues in $E$ can be expressed using $\gamma_{x}$. We can establish, for example, whether or not a set $A \in \mathcal{P}(E)$ is connected (a set $A$ is connected if and only if $A=\gamma_{x}(A), x \in A$ ), and whether or not a pair of points $x, y$ belong to the same connected component in $A$ ( $x, y$ belong to the same connected component in $A$ if and only if $x \in \gamma_{y}(A)$ or, equivalently, if and only if $\left.\gamma_{x}(A)=\gamma_{y}(A) \neq \emptyset\right)$.

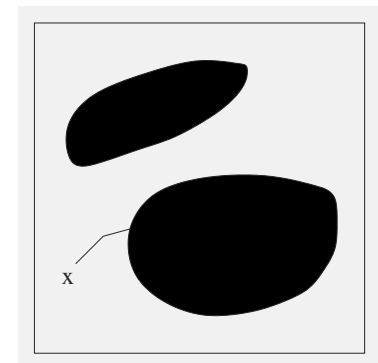

(a) Input set $A$ (in black)

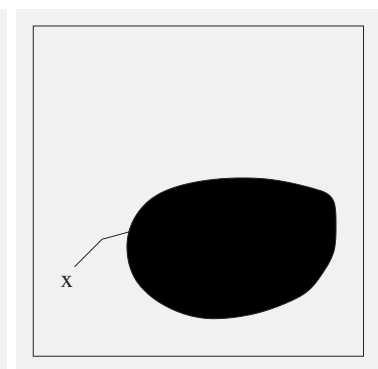

(b) $\gamma_{x}(A)$

Fig. 1. Connected component extraction. The opening $\gamma_{x}(A)$ extracts the connected component of $A$ to which $x$ belongs. 
The dual operation of $\gamma_{x}$ is the closing $\varphi_{x}$ that is equal to $E \backslash \gamma_{x} \complement(A)$, for all $A \in \mathcal{P}(E)$, where $\backslash$ denotes set difference and $\complement$ symbolizes the set complementation operator.

\subsection{Connected Operators and Connected Component Locality}

After establishing the connected class concept in the previous section, let us define the concept of connected operator. [7] [8] [9] [10].

- A connected operator only extends the input image flat zones (or piecewiseconstant regions).

It should be noticed that, from its definition, a connected operator cannot introduce discontinuities and, therefore, preserves shapes. In a binary framework, connected operators are grain-removing and pore-filling operations. Connected filters are connected operators that are idempotent.

The fact that connected filters preserve shapes makes them useful tools for image simplification and segmentation purposes 11] 12.

The concept of connected-component (c.c.) local operator, which is defined next, embraces both increasing and non-increasing operators that treat each grain and pore independently of the rest of the input [13].

Definition 1 Let $E$ be a space equiped with $\gamma_{x}$. An operator $\psi: \mathcal{P}(E) \longrightarrow \mathcal{P}(E)$ is said to be connected-component local (or c.c. local) if and only if, $\forall A \in$ $\mathcal{P}(E), \forall x \in E$

(a) $\gamma_{x}(A) \neq \emptyset, \gamma_{x} \psi(A)=\emptyset \Rightarrow \forall B \in \mathcal{P}(E), \gamma_{x}(A)=\gamma_{x}(B): \gamma_{x} \psi(B)=\emptyset$.

(b) $\gamma_{x}(A)=\emptyset, \gamma_{x} \psi(A) \neq \emptyset \Rightarrow \forall B \in \mathcal{P}(E), \gamma_{x} \complement(A)=\gamma_{x} \complement(B): \gamma_{x} \psi(B) \neq \emptyset$.

\section{Connected Filters and the Strong Property}

In this section we will study the strong property and its relationship with openings and closings, in particular to their alternating sequential compositions.

The strong property [14] 2] is a robustness property satisfied by certain morphological filters.

Definition $2 A$ filter $\Psi$ is strong if and only if $\Psi=\Psi(I \bigwedge \Psi)=\Psi(I \bigvee \Psi)$

If a filter is strong then certain variations (such as noise) of the input do not cause variations in the output. Figure2 2 illustrates this concept. Let $A$ be an input set. If $\Psi$ is strong, then for all sets $B$ such that $A \bigwedge \Psi(A) \leq B \leq A \bigvee \Psi(A) \Longrightarrow$ $\Psi(A)=\Psi(B)$. Another way to state this is saying that $\Psi$ is strong if and only if $\Psi$ is both an $\bigwedge$-filter and a $\bigvee$-filter (see [14] [2] for those related concepts). Openings and closings are, respectively, anti-extensive and extensive filters that satisfy the strong property.

In the definition of this important property does not appear any connectivity reference nor $\gamma_{x}$ in the formula. Nevertheless, in practice the strong property 


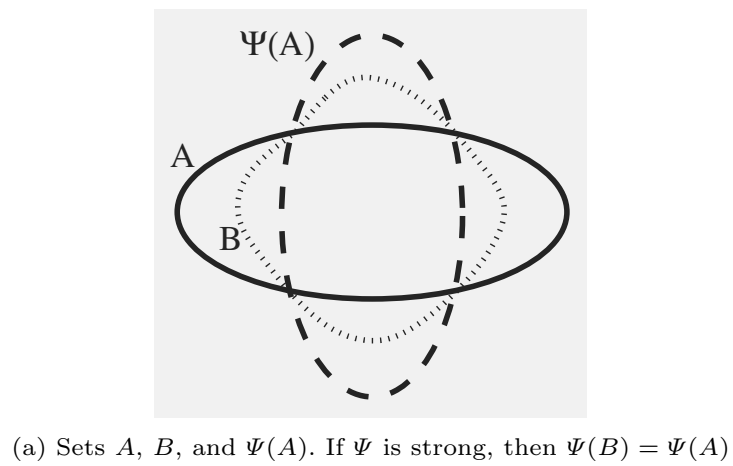

Fig. 2. Strong filter example.

is in some way quite related to connectivity, and, in particular, to connected operators. Some types of filters are not strong when they are not connected but, on the contrary, satisfy this property if they are connected. This can be the case, for example, of the connected alternating filters $\varphi \gamma$ and $\gamma \varphi$. In fact, the central point of this section will be to study the strong property under certain conditions for $\varphi$ and $\gamma$, and to use it to characterize the class of filters by reconstruction.

In some theoretical studies in mathematical morphology 2] 7] [15, the following equation

$$
\psi \gamma_{x} \psi=\gamma_{x} \psi
$$

where $\psi$ is an operator, has served to define some types of filters, especially connected filters. This interesting equation will be studied in detail in the following sections.

\subsection{Opening Case}

If $\psi$ is an opening $\gamma$ in equation (2), we have the following property:

Property $1 \gamma \gamma_{x} \gamma=\gamma_{x} \gamma \Longrightarrow \gamma$ is c.c. local.

Therefore, such an opening removes grains and treats each one independently from the rest. In fact, if an opening $\gamma$ is c.c. local and connected, then we can define $\gamma$ using the useful concept of trivial opening:

$$
\gamma=\bigvee_{x} \gamma_{\circ} \gamma_{x}
$$

where $\gamma_{\circ}$ is a trivial opening.

Definition 3 An opening $\gamma_{\circ}$ is a trivial opening [2] if 


$$
\gamma_{\circ}(A)= \begin{cases}A, & \text { if } A \text { satisfies an (increasing) criterion } \\ \emptyset, & \text { otherwise }\end{cases}
$$

Notice that the trivial opening definition does not imply c.c. locality. In fact, an opening by reconstruction $\gamma$ can be computed using a non-local trivial opening (and, nevertheless, the resulting opening by reconstruction would of course be local).

Thus, for connected openings, it can be said that equation (2) is equivalent to expression (3).

\subsection{Closing Case}

A different situation arises using equation (2) when $\psi$ is a closing $\varphi$. We introduce the following property:

Property 2 A closing $\varphi$ is connected $\Longrightarrow \varphi \gamma_{x} \varphi=\gamma_{x} \varphi$.

Property 2 states that any connected closing satisfies equation (2), i.e., that grains of the closing output are invariant under the closing. A proof is relatively straightforward. Let $E$ be the space, let $\psi$ be a closing, and let $G$ be a connected component of the output $\psi(A)$, where $A$ is an input set. Then $\psi(G)$ must be equal to $\mathrm{G}$ if $\psi$ is idempotent, antiextensive and increasing. Since $\psi$ is connected, the possibilities are (assuming the space $E$ is connected): (a) $G$, (b) $\emptyset$, or (c) $E$. However, (b) is not possible ( $\psi$ is antiextensive), and (c) is not possible either ( $\psi$ is increasing and idempotent). Therefore, $\psi(G)=G$.

An important aspect is that equation (2), when $\psi$ is a closing $\varphi$, does not imply that the closing $\varphi$ is c.c. local. And, therefore, a connected closing that satisfies equation (2) (in fact, from property 2, all of them do) cannot always be expressed by the dual of expression (3), which would be

$$
\varphi=\bigwedge_{x} \varphi_{\circ} \varphi_{x}
$$

where $\varphi_{\circ}$ is a trivial closing. The definition of the trivial closing follows (which is the dual of that of the trivial opening).

Definition 4 A closing $\varphi_{\circ}$ is a trivial closing [2] if

$$
\varphi_{\circ}(A)= \begin{cases}E, & \text { if } A \text { satisfies an increasing criterion } \\ A, & \text { otherwise }\end{cases}
$$

\subsection{Alternating Filters $\varphi \gamma$ and $\gamma \varphi$}

In this section we will get an important result by investigating the strong property of alternating filters using equation (21). Later, we will define the filter by reconstruction class in a way to avoid this type of situations. 
In [7] [15, it has been shown that if a connected opening $\gamma$ and a connected closing $\varphi$ satisfy equation (2) $\psi \gamma_{x} \psi=\gamma_{x} \psi$ (substituting $\gamma$ for $\psi$, and similarly for $\varphi$ ), then the alternating filter $\varphi \gamma$ has the desirable strong property. From property 2, we also can state this as follows.

Property 3 Let $\gamma$ be a connected opening that satisfies $\gamma \gamma_{x} \gamma=\gamma_{x} \gamma$, and let $\varphi$ be a connected closing (i.e., $\gamma$ and $\varphi$ satisfy equation (2)). Then, the alternating filter $\varphi \gamma$ is strong.

However, there is a problem using equation (2) to characterize connected openings and closings because it is not symmetrical, i.e., it does not treat in the same way to openings and closings. We have seen that, for openings, equation (2) means that we can express the opening as in expression (3). However, for closings, equation (2) does not imply that we can express the closing as in expression (4) (which is the dual of expression (3)).

Therefore, the following important (but somewhat undesirable) result should not surprise us excessively:

Property 4 Let $\gamma$ and $\varphi$ be, respectively, a connected opening and a connected closing that satisfy equation (2). Then, the alternating filter $\gamma \varphi$ is not necessarily strong.

Notice that property 4 does not contradict the morphological duality principle.

Let us show and study an example of a case in which $\gamma \varphi$ is not strong, under the assumptions of property 4. Let us first consider the opening and closing used in the example in Figure 3 which are computed employing reconstruction algorithms 16 . The opening by reconstruction will use as marker the result of the erosion $\varepsilon_{B}$, where $B$ is the structuring element that can be seen in Fig. 3(a), along with the input set. The opening $\gamma$ removes vertical grains of width smaller than that of $B$, and therefore $\gamma$ eliminates the small vertical grains at both sides, as shown in Fig. 3(b). The closing $\varphi$ uses as marker the dilation $\delta_{C}$ where the structuring element $C$ is composed by two particles, as can be seen in Fig. 3 (c). The closing $\varphi$ fills vertical pores of width 1 if there are vertical grains at left and right at a certain distance determined by $C$ (the distance between the central pore and the grains at each side). In Fig. $3(\mathrm{~d})$, the central pore has been filled. Both $\gamma$ and $\varphi$ satisfy equation (2), but $\gamma$ is c.c. local whereas $\varphi$ is not.

The example in Figure 4 illustrates property 4. Fig. 4(a) shows the input set (which is the same as that in Fig. 3(a)). The closing first fills the central pore in Fig. 4(b), and the subsequent opening removes the grains at the sides in

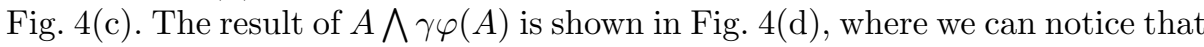
the central pore is not filled, and the grains at both sides are missing. Because the grains at both sides are missing, in Fig. 4(e) the result of the subsequent closing does not fill the central pore. Then, in Fig. U(f) the last opening leaves the image unchanged, and the final result $\gamma \varphi(A \wedge \gamma \varphi(A))$ is shown. We see clearly that $\gamma \varphi(A \bigwedge \gamma \varphi(A)) \neq \gamma \varphi(A)$, and that, therefore, $\gamma \varphi$ is not strong.

The reason to obtain this result, i.e., that $\varphi \gamma$ is strong whereas $\gamma \varphi$ is not, when $\gamma$ and $\varphi$ are connected and satisfy equation (2), is because of the nonsymmetrical nature of equation (2). 


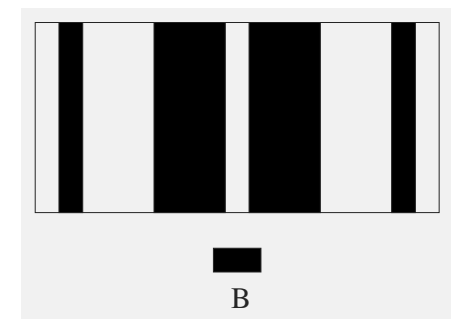

(a) Input set $A$ (in black) and stelt $B$ underneath

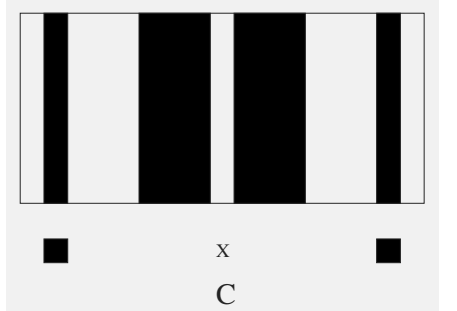

(c) Input set $A$ (in black) and stelt $C$ underneath

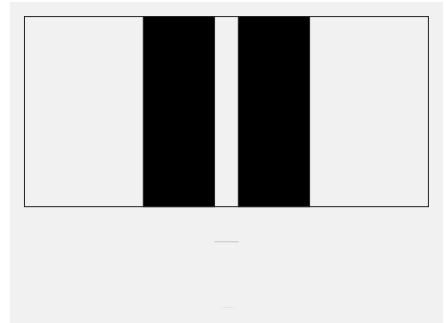

(b) $\gamma(A)$

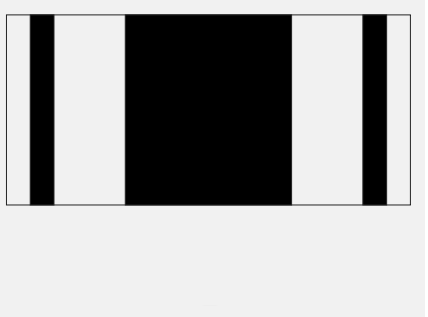

(d) $\varphi(A)$

Fig. 3. Example of results of opening $\gamma$ and closing $\varphi$. Note: the structuring elements $B$ and $C$ are both centered.

\subsection{Openings and Closings by Reconstruction, and the Filter by Reconstruction Class}

The "negative" result of property 4 strongly supports that classes of connected openings and closings be defined by dual expressions. An important class should be that defined by expresions (3) and (4).

The authors of this paper have used in other works [9] [13] 17] expresions (3) and (4) to define the important class of openings and closings by reconstruction. Since those expressions are dual of each other, openings and closings are not considered differently.

Definition 5 An opening $\gamma$ is an opening by reconstruction if and only if $\gamma=$ $\bigvee_{x} \gamma_{\circ} \gamma_{x}$, where $\gamma_{\circ}$ is a trivial opening.

Definition 6 A closing $\varphi$ is a closing by reconstruction if and only if $\varphi=$ $\bigwedge_{x} \varphi_{\circ} \varphi_{x}$, where $\varphi_{\circ}$ is a trivial closing.

The duality of the previous definitions eliminates problems such as that commented in property 4 , and we have that: If $\gamma$ and $\varphi$ satisfy, respectively, definitions 5 and 6, then $\varphi \gamma$ is strong and, by duality, $\gamma \varphi$ is strong as well. We then normally use the term filters by reconstruction to denote those filters composed of openings and closings by reconstruction. Sometimes filters by reconstruction (in particular, openings and closings by reconstuction) are defined in terms of 


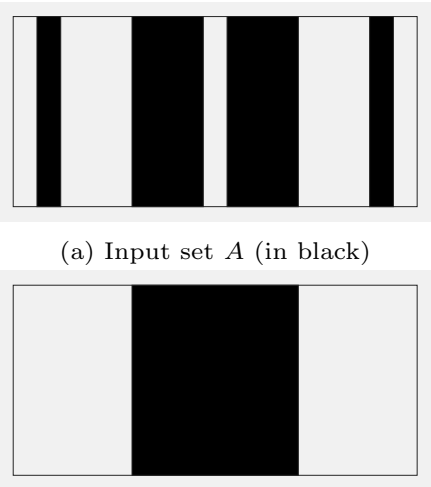

(c) $\gamma \varphi(A)$

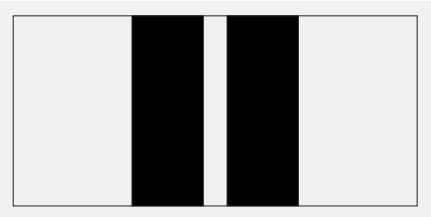

(e) $\varphi(A \wedge \gamma \varphi(A))$

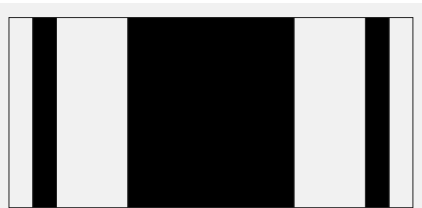

(b) $\varphi(A)$

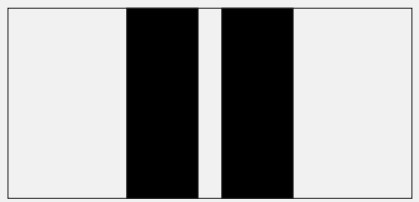

(d) $A \wedge \gamma \varphi(A)$

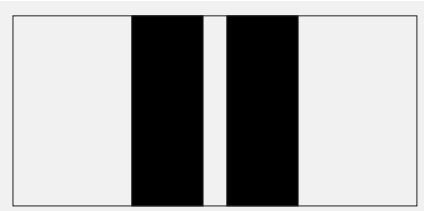

(f) $\gamma \varphi(A \wedge \gamma \varphi(A))$

Fig. 4. Strong property not satisfied. Since $\gamma \varphi(A)$ (in (c)) is different from $\gamma \varphi(A \bigwedge \gamma \varphi(A))$ (in (f)), then $\gamma \varphi$ is not strong. Note: the opening $\gamma$ and closing $\varphi$ used are those described in Fig. 3.

algorithms by reconstruction, which is an alternative and valid way to define them if the definition indicates what the markers used in the reconstruction are (in general, algorithms by reconstruction just guarantee the connectedness of the resulting operator). Nevertheless, we prefer definitions 5 and 6 since they are not linked to any particular implementation.

\section{Conclusions}

This paper has investigated the strong property of connected morphological filters, in particular of connected openings, closings and alternating filters. We have first studied a well known condition that guarantees that the connected components of a filter output are also invariant. This condition does not treat symmetrically openings and closings, and we have obtained a result in which a property satisfied by a filter is not satisfied by the dual family under that condition (a result that of course does not stand in contradiction to the morphological duality principle). This result supports that the class of openings and closings by reconstruction should be defined by dual formulae, which prevents results such as that commented in the paper. 
Acknowledgements. This work has been supported in part by "Fondo de Investigación Sanitaria" (Spanish Ministry of Health) and by "Sociedad Española de Radiología Médica".

\section{References}

1. Serra, J.: Mathematical Morphology. Volume I. London: Academic Press (1982)

2. Serra, J., ed.: Mathematical Morphology. Volume II: theoretical advances. London: Academic Press (1988)

3. Matheron, G.: Random Sets and Integral Geometry. New York: Wiley (1975)

4. Heijmans, H.: Morphological Image Operators (Advances in Electronics and Electron Physics; Series Editor: P. Hawkes). Boston: Academic Press (1994)

5. Birkhoff, G.: Lattice Theory. American Mathematical Society, Providence (1984)

6. Maragos, P., Schafer, R.: Morphological filters - part I: Their set-theoretic analysis and relations to linear-shift-invariant filters. IEEE Trans. Acoust. Speech Signal Processing 35 (1987) 1153-1169

7. Serra, J., Salembier, P.: Connected operators and pyramids. In: Proceedings of SPIE, Non-Linear Algebra and Morphological Image Processing, San Diego. Volume 2030. (1993) 65-76

8. Crespo, J., Serra, J., Schafer, R.: Image segmentation using connected filters. In Serra, J., Salembier, P., eds.: Workshop on Mathematical Morphology. (1993) $52-57$

9. Crespo, J., Serra, J., Schafer, R.: Theoretical aspects of morphological filters by reconstruction. Signal Processing 47 (1995) 201-225

10. Heijmans, H.: Connected morphological operators for binary images. Computer Vision and Image Understanding 73 (1999) 99-120

11. Crespo, J., Schafer, R., Serra, J., Gratin, C., Meyer, F.: The flat zone approach: A general low-level region merging segmentation method. Signal Processing 62 (1997) 37-60

12. Crespo, J., Maojo, V.: Shape preservation in morphological filtering and segmentation. In: XII Brazilian Symposium on Computer Graphics and Image Processing, IEEE Computer Society Press, SIBGRAPI 99. (1999) 247-256

13. Crespo, J., Schafer, R.: Locality and adjacency stability constraints for morphological connected operators. Journal of Mathematical Imaging and Vision 7 (1997) 85-102

14. Matheron, G.: Filters and lattices. In Serra, J., ed.: Mathematical Morphology Volume II: theoretical advances. London: Academic Press (1988) 115-140

15. Salembier, P., Serra, J.: Flat zones filtering, connected operators, and filters by reconstruction. IEEE Transactions on Image Processing 4 (1995) 1153-1160

16. Soille, P.: Morphological Image Analysis: Principles And Applications. SpringerVerlag Berlin, Heidelberg, New York (1999)

17. Crespo, J., Maojo, V.: New results on the theory of morphological filters by reconstruction. Pattern Recognition 31 (1998) 419-429 